\title{
Biomarker analysis of the GATSBY study of trastuzumab emtansine versus a taxane in previously treated HER2-positive advanced gastric/ gastroesophageal junction cancer
}

\author{
Manish A. Shah ${ }^{1,10}$. Yoon-Koo Kang ${ }^{2} \cdot$ Peter C. Thuss-Patience ${ }^{3} \cdot$ Atsushi Ohtsu $^{4}$. Jaffer A. Ajani ${ }^{5}$. \\ Eric Van Cutsem ${ }^{6} \cdot$ Silke Hoersch $^{7} \cdot$ Marie-Laurence Harle-Yge ${ }^{8} \cdot$ Sanne Lysbet de Haas ${ }^{9}$
}

Received: 12 October 2018 / Accepted: 26 December 2018 / Published online: 31 January 2019

(c) The International Gastric Cancer Association and The Japanese Gastric Cancer Association 2019

\begin{abstract}
Background Prespecified exploratory biomarker analyses of the phase II/III GATSBY study (NCT01641939) assessed whether patient subgroups experienced a survival benefit from trastuzumab emtansine (T-DM1) versus taxane therapy, and to advance understanding of HER2-positive advanced gastric/gastroesophageal junction cancer (AGC) disease biology. Methods Adults with HER2-positive AGC whose disease progressed during/after first-line therapy were enrolled and randomized to receive T-DM1 [Stage 1:3.6 mg/kg q3w, $2.4 \mathrm{mg} / \mathrm{kg} \mathrm{qw}$, or taxane (docetaxel/paclitaxel); Stage 2: $2.4 \mathrm{mg} / \mathrm{kg}$ qw or taxane]. Primary efficacy endpoint was overall survival (OS). Prespecified exploratory biomarkers included HER2, HER3, PTEN, PIK3CA mutation status, Fc $\gamma$ R, and cMET. Tumor samples from patients who received $2.4 \mathrm{mg} / \mathrm{kg}$ T-DM1 $(n=228)$ or taxane $(n=117)$ were included.

Results Median OS was longer in subgroups with HER2 immunohistochemistry (IHC) $3+$ [9.5 versus 8.3 months for T-DM1 versus taxane; hazard ratio (HR) 0.99 (95\% CI 0.68-1.43)] versus HER2 IHC 2+/in situ hybridization-positive [5.2 versus 9.2 months for T-DM1 versus taxane; HR 1.53 (95\% CI 0.94-2.50)] tumors. Trends towards increased median OS were also observed in subgroups with $>$ versus $\leq$ median HER2 mRNA expression, higher versus lower HER2 gene copy number, HER2 gene ratio and $\mathrm{H}$ score, and homogenous or nonfocal HER2 IHC staining. T-DM1 was not associated with superior OS versus taxane in any subgroup.

Conclusions Patients with previously treated HER2-positive AGC with higher HER2 expression experienced a better treatment effect from T-DM1 than those with lower HER2 expression and may derive comparable survival benefits from T-DM1 and taxane therapy.

Clinical trials registration NCT01641939 (https://clinicaltrials.gov/ct2/show/NCT01641939).
\end{abstract}

Keywords Trastuzumab emtansine $\cdot$ Gastric cancer $\cdot$ Gastroesophageal junction $\cdot$ Biomarkers

\section{Introduction}

Trastuzumab plus chemotherapy is approved for use in patients with HER2-positive [HER2 immunohistochemistry (IHC) 3+ or IHC $2+$ and in situ hybridization (ISH)-positive] metastatic adenocarcinoma of the stomach/gastroesophageal junction

Electronic supplementary material The online version of this article (https://doi.org/10.1007/s10120-018-00923-7) contains supplementary material, which is available to authorized users.

Manish A. Shah

mas9313@med.cornell.edu

Extended author information available on the last page of the article
(GEJ) who have not received prior anticancer treatment for their metastatic disease [1]. This is now considered the standard of care, globally [2,3]. Approval was based on pivotal data from the Trastuzumab for Gastric Cancer (ToGA) study, which demonstrated that adding trastuzumab to chemotherapy (capecitabine or fluorouracil with cisplatin) significantly improved overall survival (OS) in patients with HER2-positive advanced gastric cancer (AGC) or GEJ cancer versus chemotherapy alone [median OS 13.8 (95\% CI 12-16) versus 11.1 months (95\% CI 10-13)] [4]. The overall tumor response rate in ToGA was $47.1 \%$ in a population where $77.6 \%$ of patients had HER2 IHC $3+$ or IHC 2+/ISH-positive tumors, suggesting a substantial proportion did not respond to first-line trastuzumab treatment despite HER2 overexpression [4]. The 
mediators of primary refractory or developed HER 2 resistance in gastric cancer are not yet fully understood, although preclinical investigations are ongoing [5]. Currently, no global clinical outcomes data have established a HER2-targeted second-line regimen for patients with HER2-positive AGC.

Trastuzumab emtansine (T-DM1) is an antibody-drug conjugate of trastuzumab linked to the tubulin-binding agent DM1 via a stable thioether linker [6]. T-DM1 retains trastuzumab's HER2-targeting mechanism of action [7]. T-DM1 internalization then leads to intracellular release of DM1containing catabolites that induce mitotic arrest and cell death [6, 7]. T-DM1 improved OS and progression-free survival (PFS) in patients with metastatic breast cancer (MBC) previously treated with HER2-targeted therapies [8-10], compared with the control. These survival improvements were observed across patient subgroups in exploratory biomarker analyses [11, 12].

GATSBY was an international, randomized, open-label, adaptive phase II/III study to evaluate T-DM1 versus a taxane in patients with previously treated HER2-positive locally advanced or metastatic gastric/GEJ adenocarcinoma [13]. GATSBY did not meet its primary endpoint of improved OS in patients treated with T-DM1 $2.4 \mathrm{mg} / \mathrm{kg}$ every week (qw), compared with taxane therapy [13].

Evaluation of HER2 expression and downstream signaling will improve our understanding of HER2-positive AGC disease biology, and why T-DM1 did not demonstrate improved OS versus taxane therapy in GATSBY. Exploratory biomarker analyses in patients with MBC suggest that subgroups with higher HER2 mRNA levels (> median) experienced a larger survival benefit from T-DM1 [11, 12]. In this biomarker analysis of GATSBY, we assessed HER2 expression and additional biomarkers: HER3 - an important heterodimerization partner enabling HER2-mediated activation of the PI3K/Akt pathway [14, 15]; PTEN expression and PIK3CA mutations - associated with constitutive activation of the PI3K/Akt pathway $[16,17]$ even in the presence of HER2 inhibition [18-20]; and cMET expression-linked with resistance to HER2-targeted therapies [21, 22]. Notably, PIK3CA mutation has been linked with reduced efficacy of HER2-targeted therapies [23, 24], but not T-DM1 [11]. $\mathrm{Fc} \gamma$ receptor $(\mathrm{Fc} \gamma \mathrm{R}) \mathrm{IIa} / \mathrm{III}$ a polymorphisms were assessed as trastuzumab binds $\mathrm{Fc} \gamma \mathrm{R}$ on immune effector cells [7]. Herein we report the results of the preplanned biomarker analysis of GATSBY.

\section{Materials and methods}

\section{Patient eligibility and study design}

The design of GATSBY (NCT01641939), inclusion/exclusion criteria, and treatment schedule have been published
[13]. Patients aged $\geq 18$ years with centrally tested HER2positive (IHC $3+$, independent of ISH status, or IHC $2+$ and ISH-positive) AGC, who progressed during or after firstline therapy were eligible [13]. Patients were randomized 2:2:1 to receive $3.6 \mathrm{mg} / \mathrm{kg}$ T-DM1 every 3 weeks ( $\mathrm{q} 3 \mathrm{w}$ ), $2.4 \mathrm{mg} / \mathrm{kg}$ T-DM1 qw, or taxane (docetaxel or paclitaxel; physician's choice) in stage 1 of the study and, following independent data monitoring committee (IDMC) review, randomized 2:1 to receive the IDMC-selected dose regimen of $2.4 \mathrm{mg} / \mathrm{kg}$ T-DM1 qw or taxane in stage 2 (dose selection based on pharmacokinetic, safety, and efficacy data) [13]. The primary endpoint was OS (time from randomization to death, regardless of the cause of death); PFS [time from randomization to first occurrence of progressive disease or death from any cause (whichever occurred first)] was a secondary endpoint. GATSBY was conducted in accordance with Good Clinical Practice and the Declaration of Helsinki. The protocol was approved at each site by the local ethics committee/institutional review board. All patients provided written informed consent.

\section{Specimen characteristics}

Primary and/or metastatic tumor samples were used for biomarker analyses. Samples were supplied as formalin-fixed paraffin-embedded tumor blocks if available, or freshly cut unstained slides (shipped within $48 \mathrm{~h}$ of cutting). After HER2 assessment, additional biomarker assays were performed according to slide availability and priority. Wholeblood samples for cytogenetic analysis were collected per study protocol and shipped on dry ice to the sponsor's Clinical Sample Operations (within $48 \mathrm{~h}$ if stored at $-20{ }^{\circ} \mathrm{C}$; monthly if stored at $-70{ }^{\circ} \mathrm{C}$ ).

\section{Assay methods}

Tissue processing and assays were performed centrally by Targos Molecular Pathology prior to unblinding of the study data. IHC analysis was used to assess the expression of HER2, PTEN, and cMET. IHC staining was performed according to the manufacturers' instructions; the antibodies and validation tests used during IHC analysis are listed in Supplementary Table 1.

HER2 protein subgroups were defined by IHC staining intensity (per scoring criteria listed in Supplementary Table 2) and H score. HER 2 IHC $2+$ and IHC $3+$ subgroups (separately and combined) were further characterized as focal, heterogenous, or homogenous according to the proportion of positively stained tumor cells $(<30 \%, 30-79 \%$, or $\geq 80 \%$, respectively).

PTEN expression subgroups were categorized according to $\leq$ or $>$ median and expression levels in the tumor versus the surrounding nontumor tissue (categorized as 
"none", “decreased", "slightly decreased", "equivalent", or "increased" as described previously) [25]. PTEN was also reported as an $\mathrm{H}$ score for the cytoplasm and nucleus, determined by the percentage of viable tumor cells in each staining category: $\geq 50 \%$ no staining ( 0$), \geq 50 \%$ weak (1), $\geq 50 \%$ moderate (2), and $\geq 50 \%$ strong (3) intensity levels; and defined as $\mathrm{H}$ score $=[1 \times(\%$ cells staining at 1$)]+(2 \times(\%$ cells staining at 2$)]+[3 \times(\%$ cells staining at 3$)]$ [26]. cMET subgroups were classified by IHC staining intensity per an internally generated algorithm (Ventana/Genentech; Ventana Medical Systems, Inc., Tucson, AZ, USA): no staining or $<50 \%$ tumor cells with any intensity (IHC 0 ); $\geq 50 \%$ tumor cells with weak or higher staining but $<50 \%$ with moderate or higher intensity (IHC $1+$ ); $\geq 50 \%$ tumor cells with moderate or higher staining but $<50 \%$ with strong intensity (IHC $2+) ; \geq 50 \%$ staining with strong intensity (IHC 3+) [27].

HER2 gene amplification was measured by ISH (INFORM HER2 Dual ISH DNA Probe Cocktail Assay; Ventana Medical Systems, Inc.) and defined as positive (HER2 gene copy number: chromosome 17 centromere ratio $\geq 2.0$ ) or negative. Additional subgroups were defined by gene copy number $(<4, \geq 4$ and $<6$, or $\geq 6)$ and gene ratio $(\geq 2$ and $<4$ or $\geq 4)$. $H E R 2$ and HER 3 mRNA levels were measured using quantitative reverse transcription polymerase chain reaction (PCR; $\operatorname{cobas}^{\circledR}$ z480 Analyzer; Roche Molecular Diagnostics, Pleasanton, CA), expressed as concentration ratios relative to the housekeeping gene $G 6 P D$, and categorized as $\leq$ median or $>$ median. PIK3CA mutation analyses were performed using allele-specific PCR ( $\operatorname{cobas}^{\circledR} \mathrm{z} 480$ Analyzer) with coverage of 17 mutations in exons 1 (R88Q), 4 (N345K), 7 (C420R), 9 (E542K, E545A, E545D, E545G, E545K, Q546E, Q546K, Q546L, and Q546R), and 20 (M1043I, H1047L, H1047R, H1047Y, and G1049R), and defined as mutated or nonmutated type per exon. A validation study in gastric cancer (GC) comparing the assay to Sanger sequencing was performed before samples were analyzed. All assays were performed according to manufacturer's instructions. Fc $\gamma \mathrm{R}$ polymorphisms (rs1801274, rs396991) were assessed on whole blood-extracted DNA using TaqMan-based real-time PCR assays according to internal procedures and classified by allele expression.

\section{Statistical analyses}

The clinical cutoff point for this analysis was June 30, 2015. Exploratory OS and PFS subgroup analyses were preplanned, without preplanned sample sizes or power calculations. Median OS and PFS were calculated using the Kaplan-Meier method. Unstratified Cox proportional hazards regression was used to estimate hazard ratios (HRs) and 95\% CIs for T-DM1 versus taxane in biomarker subgroups. Multivariate analyses using Cox proportional hazards modeling were performed to explore the potential influence of HER2 IHC $3+$ staining and randomized treatment interaction on survival. Additional variables included were: treatment arms, stratification factors (world region, prior HER2-targeted therapy, prior gastrectomy), sex, age, Eastern Cooperative Oncology Group (ECOG) performance status, visceral disease (lung or liver), primary site of disease, baseline disease measurability, and extent of disease. A second model including the same variables, plus an interaction term, was used to test for multiplicative interaction between treatment effect and HER2 IHC 3+.

Subgroup treatment effect pattern plot (STEPP) analyses were performed post hoc to evaluate associations between OS and HER2 gene copy number and mRNA as continuous variables [28].

Statistical analyses were performed using SAS software (version 9.2; SAS Institute, Cary, NC, USA). STEPP plots were created with R software. All analyses were exploratory and not hypothesis-testing.

\section{Results}

\section{Patients}

Patients were enrolled between September 2012 and February 2015, and were randomized to receive T-DM1 $2.4 \mathrm{mg} /$ $\mathrm{kg}$ qw $(n=228)$, T-DM1 $3.6 \mathrm{mg} / \mathrm{kg}$ q3w $(n=70)$, or taxane $(n=117$; Supplementary Figure 1). Biomarker analyses were conducted in the intent-to-treat (ITT) population (T-DM1 $2.4 \mathrm{mg} / \mathrm{kg}$ qw and taxane arms; see Supplementary Table 3 for demographics information). Patient biomarker levels (Table 1) and median follow-up \{17.5 [interquartile range (IQR) 12.1-23.0] and 15.4 months [IQR 9.2-18.1] in T-DM1 $2.4 \mathrm{mg} / \mathrm{kg}$ qw and taxane arms, respectively\} were similar across treatment arms [13].

\section{HER2 expression/characterization at baseline}

HER2 assessments were performed on primary tumors for 312/345 (90.4\%) patients (remaining assessments performed using samples from metastases or of unknown origin). HER2 expression subgroups were equally distributed across treatment arms (Table 1); 67.2\% (231/344) of patients had HER2 IHC $3+$ status. Focal staining was observed in $15.4 \%$ of patients (53/344; Table 1); homogenous staining was observed in $56.7 \%$ of patients $(195 / 344)$, predominantly in IHC $3+$ tumors [IHC 3+: 51.1\% (118/231); IHC 2+: 16.8\% (19/113)].

HER 2 mRNA expression was similar between treatment arms (Table 1). The relationship between HER2 IHC staining and HER 2 mRNA expression is demonstrated in Fig. 1. HER 2 mRNA expression and gene ratio were numerically higher in tumors with more homogenous IHC staining. Tumors with homogenous IHC $3+$ staining had the highest median HER 2 mRNA expression and gene ratio (Fig. 1). 
Table 1 Patient biomarker levels (ITT population)

\begin{tabular}{|c|c|c|}
\hline & $\begin{array}{l}\text { T-DM1 } \\
2.4 \mathrm{mg} / \mathrm{kg} \mathrm{qw} \\
n=227^{\mathrm{a}}\end{array}$ & $n=117$ \\
\hline \multicolumn{3}{|l|}{ HER2 status [13] } \\
\hline IHC 2+/ISH-positive & $78(34.4)$ & 35 (29.9) \\
\hline IHC 3+/ISH-positive & $125(55.1)$ & $70(59.8)$ \\
\hline IHC 3+/ISH-negative & $6(2.6)$ & $2(1.7)$ \\
\hline \multirow[t]{3}{*}{ IHC 3+/ISH-unknown } & $18(7.9)$ & $10(8.5)$ \\
\hline & $\begin{array}{l}\text { T-DM1 } \\
2.4 \mathrm{mg} / \mathrm{kg} \mathrm{qw}\end{array}$ & Taxane \\
\hline & $n=228$ & $n=116$ \\
\hline \multicolumn{3}{|l|}{ HER2 staining pattern } \\
\hline Focal $(<30 \%)$ & $36(15.8)$ & $17(14.7)$ \\
\hline Heterogenous (30-79\%) & $61(26.8)$ & $35(30.2)$ \\
\hline \multirow[t]{3}{*}{ Homogenous $(\geq 80 \%)$} & $131(57.5)$ & $64(55.2)$ \\
\hline & $\begin{array}{l}\text { T-DM1 } \\
2.4 \mathrm{mg} / \mathrm{kg} \mathrm{qw}\end{array}$ & Taxane \\
\hline & $n=215$ & $n=111$ \\
\hline \multicolumn{3}{|l|}{$H E R 2 \mathrm{mRNA}^{\mathrm{b}}$} \\
\hline \multirow[t]{3}{*}{ Median $(\text { range })^{\mathfrak{c}}$} & $3.44(0.2-114.6)$ & $4.26(0.3-82.7)$ \\
\hline & $\begin{array}{l}\text { T-DM1 } \\
2.4 \mathrm{mg} / \mathrm{kg} \mathrm{qw}\end{array}$ & Taxane \\
\hline & $n=216$ & $n=111$ \\
\hline \multicolumn{3}{|l|}{$H E R 3 \mathrm{mRNA}^{\mathrm{a}}$} \\
\hline \multirow[t]{3}{*}{ Median (range) ${ }^{\mathrm{c}}$} & $0.71(0.1-12.8)$ & $0.73(0.1-6.7)$ \\
\hline & $\begin{array}{l}\text { T-DM1 } \\
2.4 \mathrm{mg} / \mathrm{kg} \mathrm{qw}\end{array}$ & Taxane \\
\hline & $n=186$ & $n=97$ \\
\hline \multicolumn{3}{|l|}{ PTEN status ${ }^{\mathrm{d}}$} \\
\hline None & $23(12.4)$ & $8(8.2)$ \\
\hline Decreased & $111(59.7)$ & $64(66.0)$ \\
\hline Slightly decreased & $36(19.4)$ & $19(19.6)$ \\
\hline Equivalent/Increased & $16(8.6)$ & $6(6.2)$ \\
\hline \multicolumn{3}{|l|}{ PTEN expression } \\
\hline$\leq$ Median & $95(51.1)$ & $52(53.6)$ \\
\hline$>$ Median & $91(48.9)$ & $45(46.4)$ \\
\hline \multicolumn{3}{|l|}{ PTEN H score } \\
\hline Mean (SD) & $121.16(72.99)$ & $118.40(72.25)$ \\
\hline \multirow[t]{3}{*}{ Median } & 100 & 100 \\
\hline & $\begin{array}{l}\text { T-DM1 } \\
2.4 \mathrm{mg} / \mathrm{kg} \mathrm{qw}\end{array}$ & Taxane \\
\hline & $n=215$ & $n=112$ \\
\hline \multicolumn{3}{|l|}{ PIK3CA mutation status } \\
\hline Mutated $^{\mathrm{e}}$ & $10(4.7)$ & $7(6.3)$ \\
\hline Exon 1 mutation $(R 88 Q)$ & $1(0.5)$ & 0 \\
\hline Exon 4 mutation (N345K) & 0 & 0 \\
\hline Exon 7 mutation $(C 420 R)$ & 0 & 0 \\
\hline Exon 9 mutation $($ E542K, E545X, Q546X) & $9(4.2)$ & $4(3.6)$ \\
\hline Exon 20 mutation (M1043I, H1047X, G1049R) & 0 & $3(2.7)$ \\
\hline
\end{tabular}


Table 1 (continued)

\begin{tabular}{|c|c|c|}
\hline & T-DM1 & Taxane \\
\hline & $n=215$ & $n=112$ \\
\hline \multirow[t]{3}{*}{ Non-mutated } & 205 (95.3) & $105(93.8)$ \\
\hline & $\begin{array}{l}\text { T-DM1 } \\
2.4 \mathrm{mg} / \mathrm{kg} \mathrm{qw}\end{array}$ & Taxane \\
\hline & $n=174$ & $n=93$ \\
\hline \multicolumn{3}{|l|}{ cMET status } \\
\hline IHC 0 & $1(0.6)$ & $6(6.5)$ \\
\hline IHC 1+ & $101(58.0)$ & $53(57.0)$ \\
\hline IHC 2+ & $57(32.8)$ & $23(24.7)$ \\
\hline \multirow[t]{3}{*}{ IHC 3+ } & $15(8.6)$ & $11(11.8)$ \\
\hline & $\begin{array}{l}\text { T-DM1 } \\
2.4 \mathrm{mg} / \mathrm{kg} \mathrm{qw}\end{array}$ & Taxane \\
\hline & $n=205$ & $n=96$ \\
\hline \multicolumn{3}{|l|}{ Fc $\gamma$ RIIa } \\
\hline $\mathrm{HH}$ & $71(34.6)$ & $41(42.7)$ \\
\hline RH & $97(47.3)$ & $36(37.5)$ \\
\hline \multirow[t]{3}{*}{$\mathrm{RR}$} & $37(18.0)$ & $19(19.8)$ \\
\hline & $\begin{array}{l}\text { T-DM1 } \\
2.4 \mathrm{mg} / \mathrm{kg} \mathrm{qw}\end{array}$ & Taxane \\
\hline & $n=206$ & $n=97$ \\
\hline \multicolumn{3}{|l|}{ Fc $\gamma$ RIIIa } \\
\hline FF & $105(51.0)$ & $50(51.5)$ \\
\hline VF & $84(40.8)$ & $38(39.2)$ \\
\hline VV & $17(8.3)$ & $9(9.3)$ \\
\hline
\end{tabular}

Data are $n(\%)$ unless otherwise stated. Differences in sample size for different biomarkers are due to availability of tissue and assay failures cMET cellular mesenchymal-epithelial transition, HER2 human epidermal growth factor receptor 2, IHC immunohistochemistry, ISH in situ hybridization, ITT intent-to-treat, $P I 3 K$ phosphoinositide 3-kinase, PIK3CA phosphatidylinositol-4,5-bisphosphate 3-kinase, catalytic subunit-alpha, PTEN phosphatase and tensin homolog, $q R T-P C R$ quantitative reverse transcription-polymerase chain reaction, $q w$ every week, $S D$ standard deviation, $T-D M 1$ trastuzumab emtansine

${ }^{a}$ One patient was randomized prior to central HER2 testing (major protocol violation reported) and was later classified as HER2-negative (IHC 0/ISH-positive)

${ }^{\mathrm{b}}$ HER2/HER3 mRNA values: conversion formulas between cobas ${ }^{\circledR} 4800$ (Roche Molecular Diagnostics, Pleasanton, CA) and LC2.0 applied

${ }^{\mathrm{c}}$ Median HER2/HER3 mRNA based on patient specimen analyzed and reported for HER2/HER3 mRNA by qRT-PCR

'Categorized as "slightly decreased", "decreased", or "equivalent/increased" compared with surrounding (normal) tissue [25]

ePatients can be counted in multiple subgroups of PI3K mutations

f“Other" in terms of PI3K non-mutated and PTEN status was either "slightly decreased", "decreased", or "equivalent/increased"

\section{Association between HER2 expression and OS}

In the T-DM1 arm, patients with tumors exhibiting higher HER2 expression levels had longer median OS, compared with those in lower HER2 expression subgroups (Table 2). This pattern was consistently observed across high versus low HER2 expression subgroups defined by protein level, gene copy number, gene amplification, $\mathrm{H}$ score, and staining pattern. In the taxane arm, OS did not appear as strongly linked with HER2 expression or amplification except for HER2 staining patterns in IHC $3+$ tumors, where homogenous staining was associated with longer median OS versus heterogenous and focal staining (Table 2). 


\section{Association between HER2 expression and comparative treatment efficacy}

Median OS was comparable between treatment arms in subgroups with IHC $3+$ tumors, $>$ median HER 2 mRNA, $\geq 6$ HER2 gene copy number, or $\geq 4 H E R 2$ gene ratio (Fig. 2a), and in patients with IHC $3+$ or IHC 2+/ISH-positive tumors exhibiting homogenous or nonfocal HER2 IHC staining patterns. However, 95\% CIs for HRs were overlapping for all subgroups (Fig. 2a). Kaplan-Meier curves support a link between HER2 expression and comparative treatment efficacy as measured by OS according to HER2 protein and mRNA expression (Fig. 2b, c). a

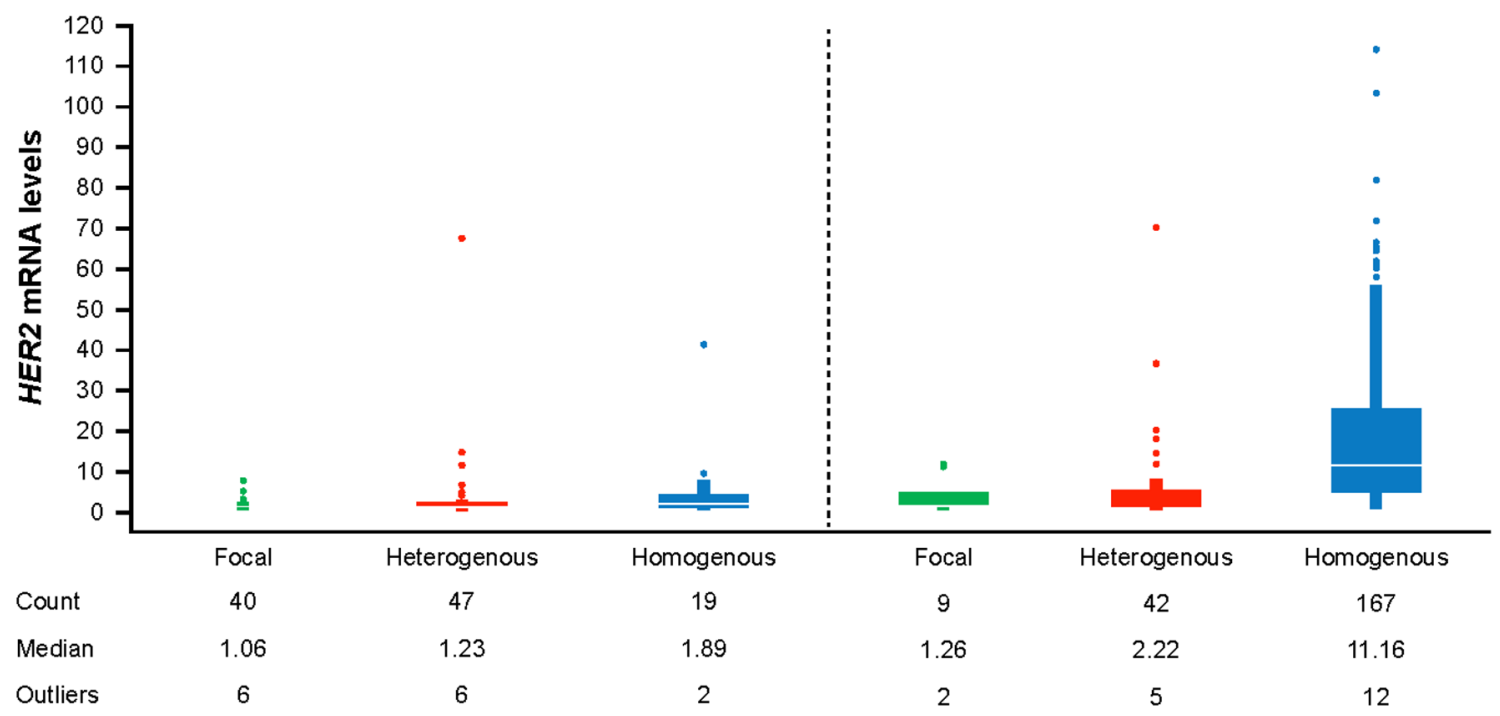

IHC 2+ and ISH-positive
IHC 3+ b

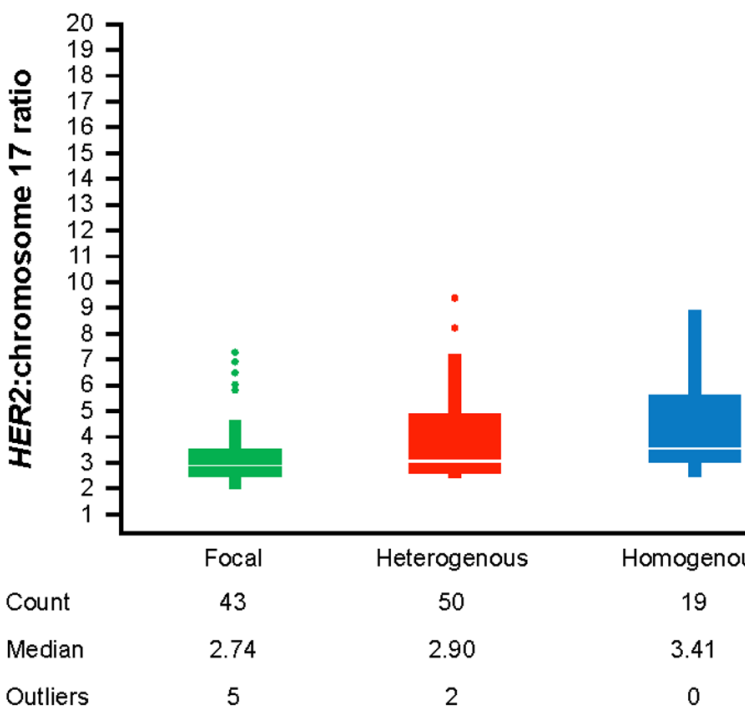

IHC 2+ and ISH-positive

IHC 3+

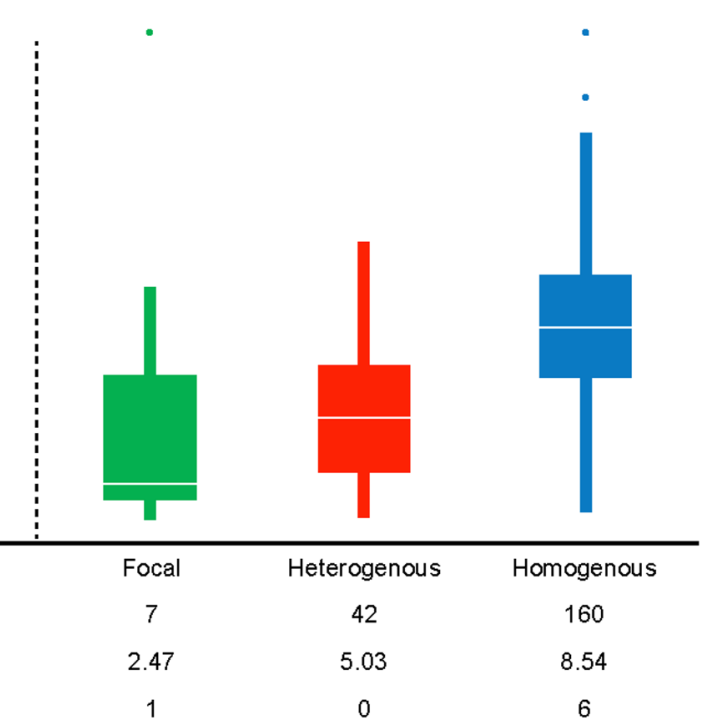

Fig. 1 HER2 expression levels in HER2 IHC 2+/ISH-positive versus IHC $3+$ subgroups (all patients): a HER2 mRNA and b HER2 gene ratio. The solid white line indicates the median, the solid blue box represents Q1-Q3, vertical blue lines extend to $1.5 \times \mathrm{IQR}$ outside the

box, and the blue dots represent individual outliers. Figures generated using Spotfire (TIBCO Software Inc., Palo Alto, CA, USA). HER2 human epidermal growth factor receptor $2, I H C$ immunohistochemistry, IQR interquartile range, $I S H$ in situ hybridization 
Table 2 Median OS in high versus low HER2 expression biomarker subgroups

\begin{tabular}{|c|c|c|c|}
\hline \multirow[t]{3}{*}{ Biomarker } & \multicolumn{3}{|c|}{ Median OS, months ( $95 \% \mathrm{CI}$ ) } \\
\hline & & $\begin{array}{l}11 \\
\mathrm{~g} / \mathrm{kg} \mathrm{qw}\end{array}$ & Taxane \\
\hline & $n$ & Median $(95 \%$ CI $)$ & Median $(95 \%$ CI) \\
\hline
\end{tabular}

HER2 protein

Low

$\begin{array}{lrlrl}\begin{array}{l}\text { IHC 2+/ISH- } \\ \text { positive }\end{array} & 79 & 5.2(4.1-7.1) & 35 & 9.2(4.7-12.8) \\ \begin{array}{l}\text { High } \\ \text { IHC 3+ }\end{array} & 149 & 9.5(8.0-11.7) & 82 & 8.5(7.1-11.7)\end{array}$

HER2 IRNA expression

Low

$\begin{array}{lrlrl}\leq \text { Median } & 108 & 6.3(5.2-7.8) & 55 & 8.5(5.5-10.0) \\ \text { High } & & & & \\ \quad>\text { Median } & 107 & 9.7(8.7-12.6) & 56 & 8.3(7.1-13.0) \\ \begin{array}{l}\text { HER2 gene copy number } \\ \text { Low }\end{array} & & & & \\ \quad<4 & 8 & 4.8(2.7-12.8) & 3 & 4.1(\mathrm{NE}) \\ \geq 4 \text { to }<6 & 35 & 4.3(3.5-6.7) & 10 & 9.4(5.5-\mathrm{NE}) \\ \text { High } & & & & \\ \geq 6 & 170 & 8.8(7.3-9.7) & 97 & 8.5(7.1-11.2)\end{array}$

HER 2 gene ratio amplification

Low

\begin{tabular}{|c|c|c|c|c|}
\hline$\geq 2$ to $<4$ & 67 & $5.2(3.9-7.0)$ & 38 & $6.5(4.2-12.7)$ \\
\hline \multicolumn{5}{|l|}{ High } \\
\hline$\geq 4$ & 139 & $9.4(7.8-10.7)$ & 70 & $8.3(7.3-11.8)$ \\
\hline \multicolumn{5}{|l|}{ H score } \\
\hline \multicolumn{5}{|l|}{ Low } \\
\hline$<$ median & 110 & $5.5(4.1-7.7)$ & 58 & $6.5(5.1-9.7)$ \\
\hline \multicolumn{5}{|l|}{ High } \\
\hline$\geq$ Median & 118 & $10.1(8.7-12.2)$ & 58 & $8.9(7.8-13.0)$ \\
\hline \multicolumn{5}{|c|}{ HER2 staining pattern: IHC $2+/ 3+$} \\
\hline \multicolumn{5}{|c|}{ Low } \\
\hline Focal $(<30 \%)$ & 36 & $4.0(2.6-5.2)$ & 17 & $6.5(3.2-\mathrm{NE})$ \\
\hline $\begin{array}{c}\text { Heterogenous } \\
(30-79 \%)\end{array}$ & 61 & $6.6(4.8-8.7)$ & 35 & $6.5(4.6-11.8)$ \\
\hline \multicolumn{5}{|l|}{ High } \\
\hline $\begin{array}{l}\text { Homogenous } \\
\quad(\geq 80 \%)\end{array}$ & 131 & $9.6(8.7-11.7)$ & 64 & $9.0(7.8-12.2)$ \\
\hline \multicolumn{5}{|c|}{ HER2 staining pattern: IHC $3+$} \\
\hline \multicolumn{5}{|l|}{ Low } \\
\hline Focal $(<30 \%)$ & 33 & $5.6(3.3-9.7)$ & 15 & $6.0(3.3-\mathrm{NE})$ \\
\hline $\begin{array}{l}\text { Heterogenous } \\
(30-79 \%)\end{array}$ & 42 & $9.2(7.1-13.1)$ & 23 & $6.1(4.6-9.7)$ \\
\hline \multicolumn{5}{|l|}{ High } \\
\hline $\begin{array}{l}\text { Homogenous } \\
(\geq 80 \%)\end{array}$ & 74 & $11.8(9.4-15.2)$ & 44 & $11.7(8.1-\mathrm{NE})$ \\
\hline
\end{tabular}

CI confidence interval, HER2 human epidermal growth factor receptor 2, IHC immunohistochemistry, ISH in situ hybridization, $N E$ not evaluable, $O S$ overall survival, $q w$ every week, $T-D M 1$ trastuzumab emtansine
Median PFS in patients with high HER2 expression was generally similar between T-DM1 and taxane treatment arms, whereas median PFS in patients with low HER2 expression (e.g. IHC 2+/ISH-positive, or low mRNA HER2 expression) indicated significantly worse survival outcomes with T-DM1, compared with taxane treatment in this population (Fig. 2d).

Post hoc multivariate Cox regression modeling suggested that HER2 IHC $3+$ staining was an independent predictor of OS (HR 0.58; $p=0.0001$; Table 3 ). The addition of an IHC $3+*$ T-DM1 treatment interaction term (model 2) confirmed that the longer OS in HER2 IHC 3+ versus IHC $2+$ subgroups specifically occurred in association with T-DM1 (Table 3). Other variables identified as independent prognostic factors for improved OS were prior gastrectomy $(p=0.0002)$, visceral disease (lung or liver) at baseline $(p=0.0314)$, and an ECOG performance status of 0 at baseline ( $p=0.0297$; Table 3). Prior HER2-targeted therapy also appeared to have an effect [HR $0.75,95 \%$ CI 0.54-1.05 (model 1) and HR 0.75, 95\% CI 0.55-1.03 (model 2), respectively]. STEPP analysis revealed no linear pattern for HER 2 mRNA, or HER 2 gene copy number when correlated with the treatment effect (HR) on OS (Supplementary Figure 2). For PFS, the STEPP analysis showed a more consistent linear pattern for HER2 mRNA and HER2 gene copy number than observed with OS (Supplementary Figure 3).

\section{Association between HER3 mRNA, PTEN, cMET expression, PIK3CA mutation status, and FcyR polymorphisms, and comparative treatment efficacy}

A reduced treatment effect was observed with T-DM1 versus taxane therapy in patients with $>$ median HER3 mRNA levels (Fig. 3). No clear differences were identified within the PTEN, cMET, and Fc $\gamma \mathrm{R}$ subgroups, although patients with high cMET protein expression (IHC $3+$ ) showed a numerically better treatment effect with T-DM1, compared with taxane therapy (Fig. 3; Supplementary Figure 4). However, no firm conclusions can be drawn given the small subgroup sizes. The patient subgroup with PIK3CA mutations was also too small $(n=17)$ to allow interpretation.

\section{Discussion}

GATSBY did not show superior OS with T-DM1 versus taxane therapy, in previously treated AGC [13]. The results from this prespecified biomarker analysis support the hypothesis that tumors with evidence of high HER2 expression, as defined by IHC $3+, H E R 2$ mRNA expression $>$ median, gene ratio $\geq 4$, or homogenous or nonfocal staining may be more sensitive to T-DM1 therapy, with survival outcomes comparable to taxane therapy. 


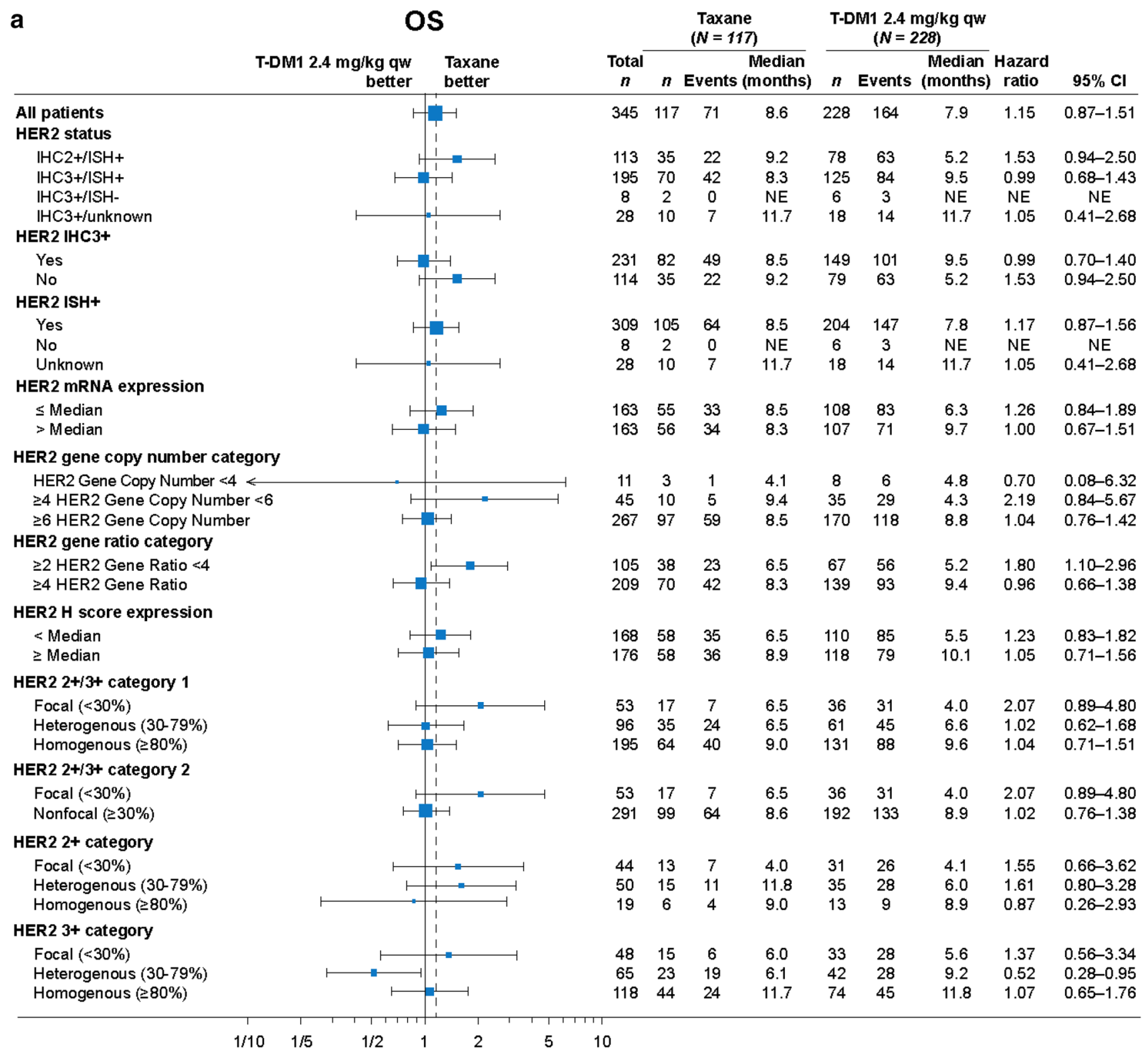

Fig. 2 Comparative efficacy of T-DM1 and taxane therapies in HER2 expression subgroups: a OS in individual subgroups and KaplanMeier curves showing treatment and biomarker effect on OS according to b HER2 expression and $\mathbf{c} H E R 2$ mRNA levels; d PFS in individual subgroups. Dotted line on forest plots represents the hazard ratio for the overall ITT population. AVAL elapsed time to the event

Comparable OS and PFS in the T-DM1 and taxane arms were observed in patients with HER2 IHC $3+$ and $>$ median HER 2 mRNA, while taxane therapy was associated with longer survival than T-DM1 in patients with lower HER2 expression. This differential treatment benefit between high and low HER2 expression was more pronounced for PFS than OS. This may result from PFS being evaluated during the GATSBY study treatment period, whereas any putative relationship between biomarkers and final OS could be of interest, $C I$ confidence interval, HER2 human epidermal growth factor receptor 2, IHC immunohistochemistry, ISH in situ hybridization, ITT intent-to-treat, $N E$ not evaluable, $O S$ overall survival, $P F S$ progression-free survival, $q w$ every week, $T-D M 1$ trastuzumab emtansine

confounded by post-progression treatments. However, perprotocol investigator assessment of PFS within an open-label study could also potentially affect the data. Interpretation of the cMET analysis was limited by the small patient numbers in the cMET IHC 0 and IHC $3+$ groups. The observed 5.2\% mutation rate for PIK3CA limited the analysis of its impact on treatment outcomes in this study, and HER2-positive GC biology (mutation rates in $\mathrm{BC}$ are substantially higher, e.g., 
b

HER2 expression IHC2 +//SH-positive vs. IHC 3+

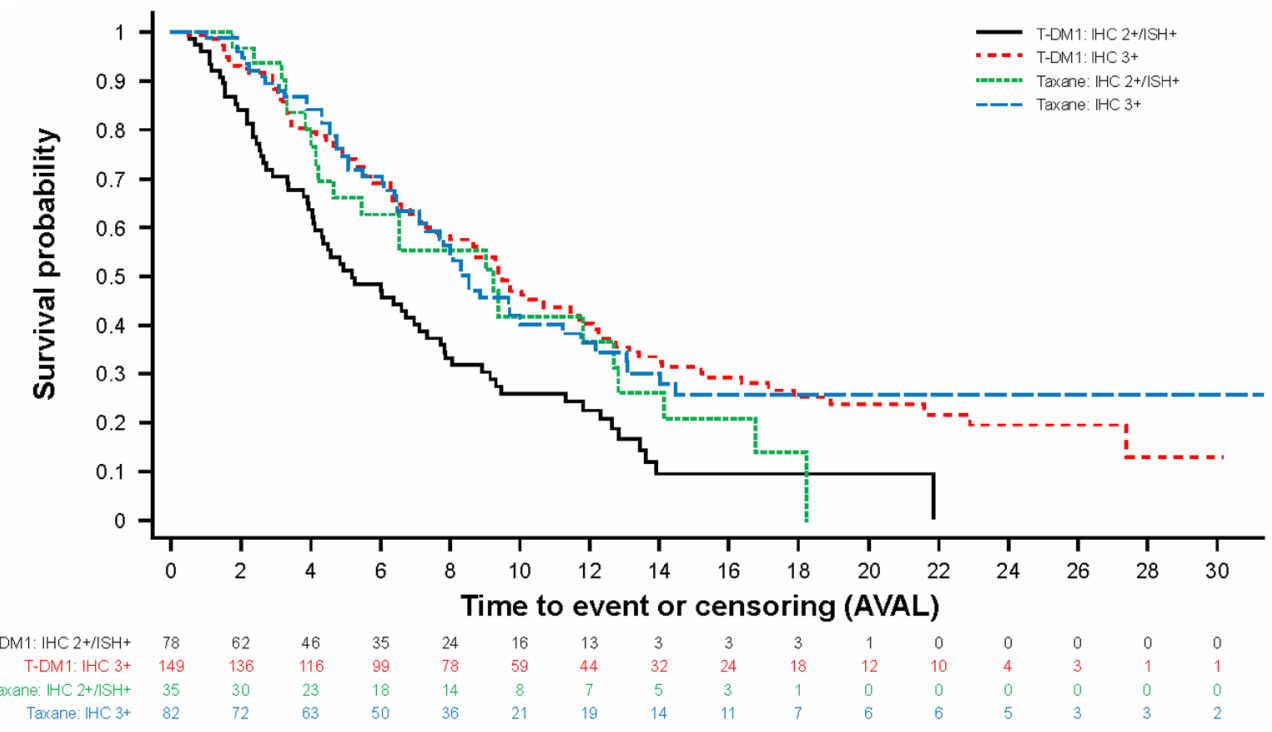

c

HER2 mRNA expression ( $\leq$ median vs. > median)

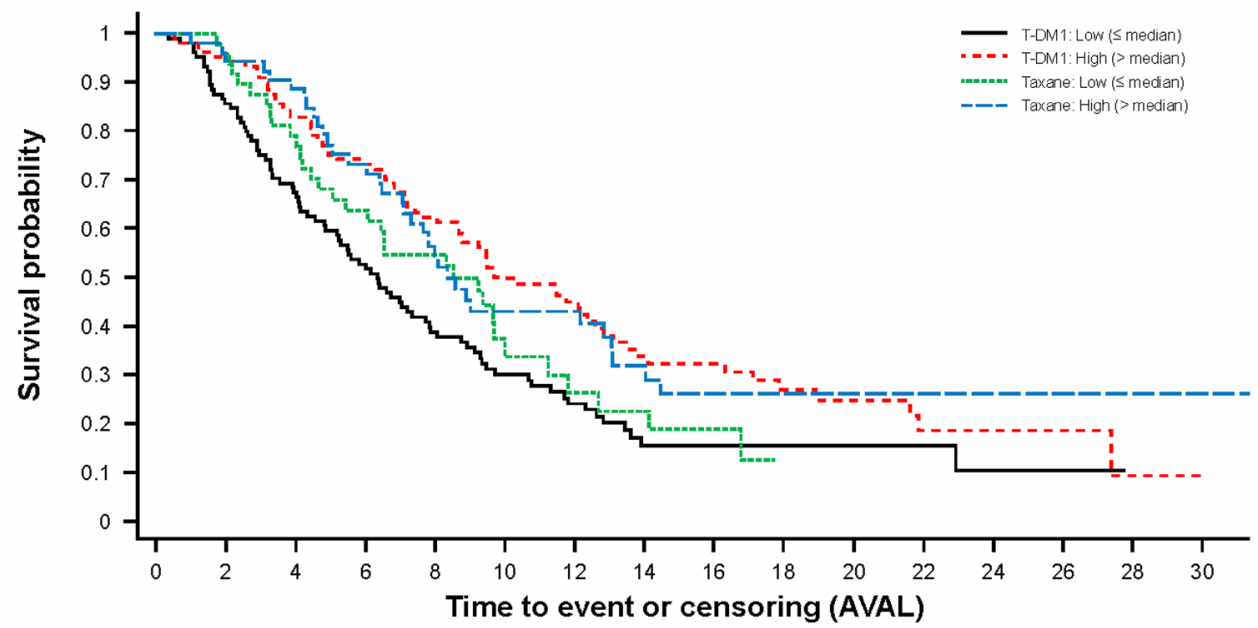

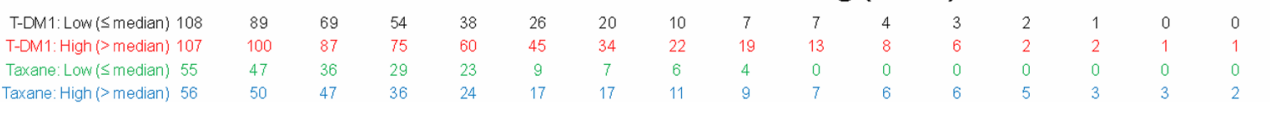

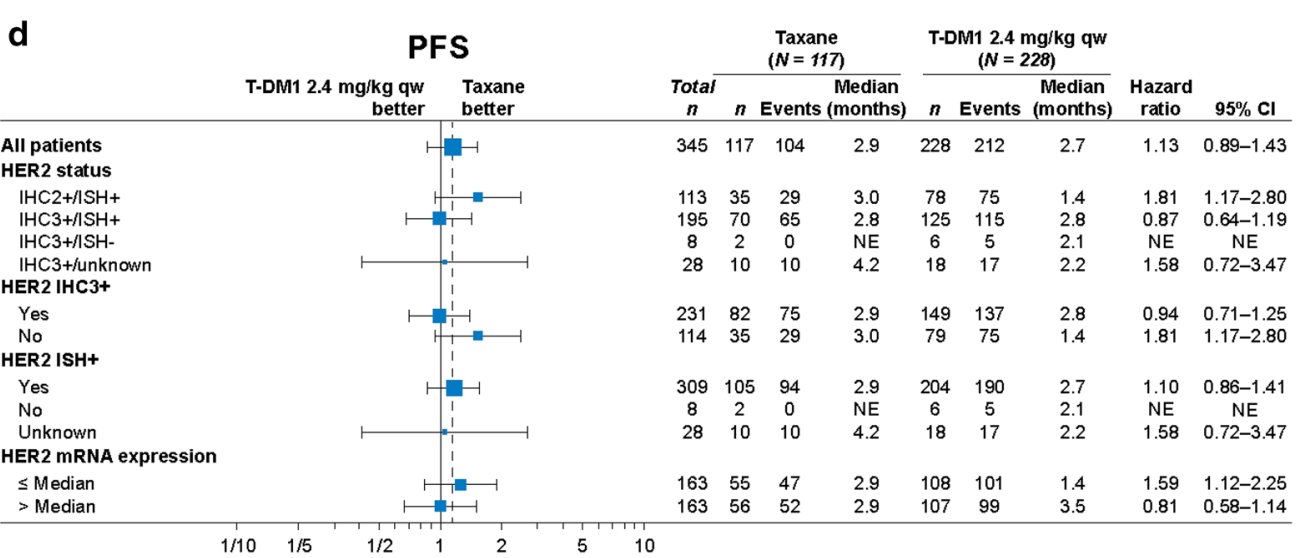

Fig. 2 (continued) 
Table 3 Multivariate analysis of OS

Model number/variables Included

$p$ value-selected independent

Comparison

$p$ value Hazard ratio $(95 \% \mathrm{CI})$ predictors $^{\mathrm{a}}$

\section{Model 1}

Treatment arms

Stratification factors ${ }^{b}$

Sex

Age

ECOG performance status

Visceral disease ${ }^{c}$

Primary site of disease

Measurability

Extent of disease $^{\mathrm{d}}$

HER2 IHC 3+ (yes versus no)

$N=345$ (235 events)

Model 2

Same as in Model 1 (not shown)

plus HER2 IHC 3+ treatment arm interaction $^{\mathrm{a}}$

$N=345$ (235 events)
Treatment

Prior HER2 therapy

Prior gastrectomy

Visceral disease

ECOG performance status

HER2 IHC 3+

$$
\begin{aligned}
& \text { T-DM1 qw }(n=228) \text { vs. taxane } \\
& (n=117)
\end{aligned}
$$

Yes $(n=267)$ vs. no $(n=78)$

$0.19681 .21(0.91-1.61)$

Yes $(n=103)$ vs. no $(n=242)$

$0.0909 \quad 0.75(0.54-1.05)$

Yes $(n=219)$ vs. no $(n=126)$

$0.0002 \quad 0.56(0.41-0.76)$

$0.0314 \quad 1.41(1.03-1.92)$

$0(n=142)$ vs. $\geq 1 \quad(n=203)$

$0.0297 \quad 0.74(0.56-0.97)$

Yes $(n=231)$ vs. no $(n=114)$

$0.0001 \quad 0.58(0.44-0.77)$

HER2 IHC 3+ Treatment arm interaction
Weekly T-DM1
Yes $(n=149)$ vs. no $(n=79)$
$0.0568 \quad 0.49(0.35-0.68)$
Taxane
Yes $(n=82)$ vs. no $(n=35)$
$0.88(0.52-1.48)$

All analyses are exploratory; no significance level was assigned

$C I$ confidence interval, ECOG Eastern Cooperative Oncology Group, HER2 human epidermal growth factor receptor 2, IHC immunohistochemistry, $O S$ overall survival, $q w$ weekly, $T-D M 1$ trastuzumab emtansine

${ }^{\mathrm{a}}$ Treatment arms and other factors with $p$ values $<0.1$ displayed

${ }^{\mathrm{b}}$ Stratification factors were world region, prior HER2-targeted therapy, and prior gastrectomy

${ }^{\mathrm{c}}$ Lung or liver disease

\begin{tabular}{|c|c|c|c|c|c|c|c|c|c|c|c|}
\hline \multirow[b]{2}{*}{ Biomarkers - mRNA } & \multicolumn{3}{|c|}{ OS } & \multicolumn{3}{|c|}{$\begin{array}{l}\text { Taxane } \\
(N=117)\end{array}$} & \multicolumn{3}{|c|}{$\begin{array}{c}\text { T-DM1 } 2.4 \mathrm{mg} / \mathrm{kg} \mathrm{qw} \\
(N=228)\end{array}$} & \multirow[b]{2}{*}{$\begin{array}{l}\text { Hazard } \\
\text { ratio }\end{array}$} & \multirow[b]{2}{*}{$95 \% \mathrm{Cl}$} \\
\hline & $\begin{array}{r}\text { T-DM1 } 2.4 \mathrm{mg} / \mathrm{kg} \mathrm{qw} \\
\text { better }\end{array}$ & \multirow[t]{2}{*}{$\begin{array}{l}\text { Taxane } \\
\text { better }\end{array}$} & $\begin{array}{c}\text { Total } \\
n\end{array}$ & \multicolumn{2}{|r|}{ Eyents } & $\begin{array}{l}\text { Median } \\
\text { (months) }\end{array}$ & \multirow{2}{*}{$\frac{n}{228}$} & \multirow{2}{*}{$\begin{array}{c}\text { Events } \\
164\end{array}$} & \multirow{2}{*}{$\begin{array}{c}\frac{\text { Median }}{\text { (months) }} \\
7.9\end{array}$} & & \\
\hline All Patients & $\mapsto$ & & 345 & 117 & 71 & 8.6 & & & & 1.15 & $0.87-1.51$ \\
\hline HER3 mRNA expression & & & & & & & & & & & \\
\hline$\leq$ Median & $\longrightarrow$ & $=$ & 167 & 56 & 36 & 8.6 & 111 & 77 & 9.2 & 0.97 & $0.65-1.45$ \\
\hline$>$ Median & & +1 & 160 & 55 & 31 & 6.5 & 105 & 78 & 7.2 & 1.27 & $0.84-1.93$ \\
\hline PTEN expression & & & & & & & & & & & \\
\hline$\leq$ Median & $\vdash$ & - & 147 & 52 & 32 & 9.2 & 95 & 74 & 7.1 & 1.20 & $0.79-1.81$ \\
\hline$>$ Median & $\longmapsto$ & + & 136 & 45 & 29 & 7.3 & 91 & 63 & 9.1 & 0.96 & $0.62-1.49$ \\
\hline PTEN status & & & & & & & & & & & \\
\hline None & $=-$ & $\stackrel{1}{1}$ & 31 & 8 & 6 & 5.5 & 23 & 19 & 6.6 & 0.81 & $0.32-2.06$ \\
\hline Decreased & $\longmapsto$ & 1 & 175 & 64 & 39 & 8.3 & 111 & 84 & 7.7 & 1.08 & $0.74-1.58$ \\
\hline Slightly decreased & & -1 & 55 & 19 & 13 & 9.7 & 36 & 25 & 9.5 & 1.13 & $0.57-2.22$ \\
\hline Equivalent/increased & & 1 & 22 & 6 & 3 & NE & 16 & 9 & 6.0 & 0.92 & $0.24-3.45$ \\
\hline PI3K subgroup & & & & & & & & & & & \\
\hline PIK $3 C A$ mutated & & 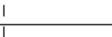 & 17 & 7 & 5 & 9.1 & 10 & 7 & 4.3 & 1.08 & $0.34-3.44$ \\
\hline Non-mutated & $\mapsto$ & -1 & 310 & 105 & 63 & 8.6 & 205 & 146 & 8.6 & 1.11 & $0.83-1.49$ \\
\hline PI3K and PTEN & & & & & & & & & & & \\
\hline PI3K mutated and/or & PTEN none & 1 & 47 & 15 & 11 & 6.5 & 32 & 26 & 5.0 & 1.08 & $0.53-2.21$ \\
\hline Other $r^{9}$ & & -1 & 229 & 80 & 49 & 8.5 & 149 & 106 & 9.1 & 1.04 & $0.74-1.45$ \\
\hline cMET status & & & & & & & & & & & \\
\hline $\mathrm{HHC1+}$ & $\longmapsto$ & $=-1$ & 154 & 53 & 34 & 9.0 & 101 & 71 & 8.7 & 1.17 & $0.77-1.76$ \\
\hline $\mathrm{IHC2+}$ & & & 80 & 23 & 11 & 9.7 & 57 & 45 & 7.0 & 1.45 & $0.75-2.81$ \\
\hline $\mathrm{IHC3+}$ & $\longmapsto$ & 4 & 26 & 11 & 11 & 4.6 & 15 & 10 & 7.8 & 0.50 & $0.21-1.19$ \\
\hline
\end{tabular}

${ }^{\mathrm{d}}$ Unresectable locally advanced/recurrent disease or metastatic disease

Fig. 3 OS in HER3 mRNA, PTEN, cMET, and PIK3CA mutation status subgroups. Dotted line represents the hazard ratio for the overall ITT population. $C I$ confidence interval, $c M E T$ cellular mesenchymalepithelial transition, HER3 human epidermal growth factor receptor
3, ITT intent-to-treat, $N E$ not evaluable, $O S$ overall survival, $P I 3 K$ phosphoinositide 3-kinase, PIK3CA phosphatidylinositol-4,5-bisphosphate 3-kinase, catalytic subunit-alpha, PTEN phosphatase and tensin homolog, $q w$ every week, $T-D M 1$ trastuzumab emtansine 
$30.5 \%$ in EMILIA [11], but can be as low as $3 \%$ in some GC subtypes [29]).

Our findings support previous observations that heterogenous HER2 staining patterns seem more common in GC than BC [30-32]. The proportion of patients in GATSBY with HER2 IHC 2+/ISH-positive tumors was similar to that reported in ToGA (32.8\% and 27\%, respectively) [4], and is markedly higher than the $\sim 10 \%$ rate observed in $\mathrm{BC}$ [33]. Median HER2 mRNA expression levels in this study were also lower than those measured in BC studies [median ranges 9-13 in BC (Roche, Data on file)].

Biomarker analyses from EMILIA and TH3RESA in patients with HER2-positive advanced/MBC reported that the statistically significant improvements in PFS and OS in patients receiving T-DM1 versus control were maintained across all subgroups examined (HER2 and HER 3 mRNA, PTEN protein and PIK3CA mutation status [11, 12], and EGFR mRNA [11]). These analyses also reported greater improvements in survival endpoints among patient subgroups with $>$ median HER2 mRNA expression [11, 12]. A similar pattern was identified in this biomarker analysis of GATSBY (although to a lesser extent), with increased PFS and OS in patients with higher versus lower levels of HER2 expression in the T-DM1 arm. Although in BC, even patients with lower HER2-expressing tumors experienced a PFS and OS benefit with T-DM1, compared with the control arm [11, 12], whereas in GATSBY T-DM1 was not associated with superior treatment outcomes versus taxane therapy in the ITT population or any biomarker subgroup.

The reasons for the lack of T-DM1 effect on OS and PFS in GATSBY are unclear, but suggest that HER2 expression could drive disease progression differently in GC versus $\mathrm{BC}$, which could have important implications for gastric tumor biology. Specifically, the malignant phenotype may be driven more by HER2 signaling in BC than in GC, leading to greater sensitivity to HER2-targeted therapies in BC. This concept is further supported by the lack of benefit of other HER2 inhibitors in GC, such as lapatinib [34, 35]. Furthermore, IHC scoring in GC allows strong incomplete membrane staining to be classified as HER2-positive [31]. This could mean HER2 receptor density is lower in GC than BC even in homogenously stained tumors; comparison of HER2 mRNA levels between GC and BC tumors with homogenous HER2 staining does not support this hypothesis (Roche, Data on file); however, more accurate methods to measure exact HER2 receptor density per cell may be needed to test this assumption. Discordance in HER2 expression between primary and metastatic GC has been reported previously, suggesting that HER 2 status can be altered or lost during disease progression [36, 37]; HER2 status was confirmed using a primary tumor sample for approximately $90 \%$ of patients in GATSBY (all had status confirmed using tissue collected prior to first-line therapy). HER2 expression could also have changed before versus after first-line HER2-targeted therapy [38], and in primary versus metastatic tumors during disease progression. However, our multivariate analysis of OS, and the previously published subgroup analysis for the GATSBY study, does not indicate a worse outcome in patients in the T-DM1 arm who received prior HER2-targeted therapy [13]. There could also be non-HER2-mediated, as yet unknown, resistance mechanisms in GC that influenced the treatment outcomes in this study.

The TRIO-013/LOGiC study (lapatinib plus chemotherapy as first-line therapy for patients with upper gastrointestinal adenocarcinomas), reported lapatinib was associated with improved PFS in those with metastatic cancers with high-level HER2 amplification [34]. Subgroup analyses of the TyTAN study (lapatinib plus paclitaxel as second-line therapy in patients with AGC) also reported that, although HER2-targeted therapy failed to improve OS versus paclitaxel alone in the ITT population, HER2 IHC $3+$ patients who received lapatinib plus paclitaxel had a significantly reduced risk of death and PFS [35]. However, there are important differences between the study populations of TyTAN and GATSBY: TyTAN enrolled only Asian patients, whereas patients from Asia-Pacific comprised $46 \%$ of the GATSBY population; only $6 \%$ in TyTAN had previously received trastuzumab, compared with $77.4 \%$ in GATSBY $[13,35]$.

This analysis provides the first large-scale formal evaluation of predefined biomarkers in HER2-positive AGC. The population was representative of patients with HER2-positive AGC who had relapsed or progressed during or after first-line therapy. However, the study was powered for the primary efficacy endpoint and not for biomarker analyses, and only a limited panel of biomarkers was assessed. The PFS data could be confounded by the open-label study design and investigator assessment. Additionally, the primary tumor tissue analyzed for most patients might not reflect the tumor biology, including HER2 status, at study entry.

In this exploratory biomarker analysis of GATSBY, patients with high HER2 expression (as reflected by several HER 2 markers) had a comparable OS and PFS benefit from T-DM1 as from taxanes; although T-DM1 $2.4 \mathrm{mg} / \mathrm{kg}$ weekly did not show superiority over taxanes for OS and PFS in any of the prespecified biomarker subgroups of patients with previously treated HER2-positive AGC. The analyses presented advance the understanding of HER2 biology in gastric tumors; however, to improve future studies of HER2targeted agents in GC, it may be useful to implement mandatory collection of tumor tissue at study entry and focus enrollment on patients with tumors that show high HER2 expression levels and/or a more homogenous HER2 IHC staining pattern. 
Acknowledgements We would like to acknowledge all patients and investigators participating in this trial and the central testing and biomarker laboratories (Targos Molecular Pathology GmbH, Kassel, Germany; Aros Applied Biotechnology A/S, Aarhus, Denmark; Genentech, Inc., BRM Operations Handling, South San Francisco, CA). We also gratefully acknowledge the contributions of Tina van Der Horst to all data analyses, and of Dr. Marjorie Green to the design and conduct of the study. Support for third-party writing assistance for this manuscript, furnished by Debbie Briggs, Ph.D., and Rachel Johnson, Ph.D., of Health Interactions, was provided by F. Hoffmann-La Roche Ltd, Basel, Switzerland.

Author contributions Conception and design: MAS, PCT-P, SLH, JAA, M-LH-Y. Acquisition of data: MAS, Y-KK, PCT-P, EC, SLH, M-LH-Y. Analysis and interpretation of data: MAS, Y-KK, PCT-P, AO, EC, SLH, SH, M-LH-Y. Writing, review, and/or revision of the manuscript: MAS, Y-KK, PCT-P, AO, JAA, EC, M-LH-Y, SLH, SH. Administrative, technical, or material support (i.e., reporting or organizing data, constructing databases): Study supervision: MAS

Funding The sponsor of the GATSBY study was F. HoffmannLa Roche Ltd, Basel, Switzerland. F. Hoffmann-La Roche Ltd was involved in the study design, the data interpretation, and the decision to submit for publication in conjunction with the authors.

\section{Compliance with ethical standards}

Disclosure of prior publication/presentation This exploratory biomarker analysis has not been presented previously. Selected baseline data and univariate analyses have been published in the primary analysis: Thuss-Patience et al. [13].

Conflict of interest Manish A. Shah has received research funding from Roche, Boston Biomedical, and Merck. Yoon-Koo Kang has acted in a consulting or advisory role for Novartis, Roche, BMS, Ono, Daehwa, and Blueprint and has received research funding from Daehwa. Peter C. Thuss-Patience has acted in a consulting or advisory role for Roche, Lilly, Pfizer, MSD, BMS, and Nordic and has received research funding (institutional support) from GSK and Novartis, and travel, accommodation, and expenses from Roche, Merck-KGaA, and TEVA. Atsushi Ohtsu has received research funding from BMS and a family member is employed by Celgene. Jaffer A. Ajani has received honoraria from BMS, Merck, Lilly, Taiho, and Astellas, has acted in a consulting or advisory role for Lilly, BMS, Insys, and AstraZeneca Medpace, and has received research funding from Merck, Delta-Fly, Gilead, Roche, and BMS. Eric Van Cutsem has acted in a consulting or advisory role for Bayer, Lilly, Roche, and Servier, and has received research funding (institutional support) from Amgen, Bayer, Boehringer, Lilly, Novartis, Roche, Sanofi, Celgene, Servier, Merck-KGaA, Merck, and Ipsen. Silke Hoersch is employed by KOEHLER eClinical and F. Hoffmann-La Roche. Marie-Laurence Harle-Yge is employed by F. Hoffmann-La Roche. Sanne Lysbet de Haas is employed by F. Hoffmann-La Roche.

Human rights statement GATSBY was conducted in accordance with Good Clinical Practice and the Declaration of Helsinki. The protocol was approved at each site by the local ethics committee/institutional review board. All patients provided written informed consent.

\section{References}

1. Roche Registration Ltd. Herceptin ${ }^{\circledR}$ (trastuzumab). Summary of product characteristics. http://www.ema.europa.eu/docs/en_GB/ document_library/EPAR_-_Product_Information/human/00027 8/WC500074922.pdf. Accessed Sept 2018.

2. Waddell T, Verheij M, Allum W, Cunningham D, Cervantes A, Arnold D, et al. Gastric cancer: ESMO-ESSO-ESTRO Clinical Practice Guidelines for diagnosis, treatment and follow-up. Ann Oncol. 2013;24(Suppl. 6):vi57-63.

3. National Comprehensive Cancer Network (NCCN). NCCN Clinical Practice Guidelines in Oncology (NCCN Guidelines ${ }^{\circledR}$ ): Gastric Cancer. Version 3. 2016. http://www.nccn.org/professionals/physi cian_gls/pdf/gastric.pdf. Accessed Aug 2016.

4. Bang YJ, Van Cutsem E, Feyereislova A, Chung HC, Shen L, Sawaki A, et al. Trastuzumab in combination with chemotherapy versus chemotherapy alone for treatment of HER2-positive advanced gastric or gastro-oesophageal junction cancer (ToGA): a phase 3, open-label, randomised controlled trial. Lancet. 2010;376:687-97.

5. Yang Z, Guo L, Liu D, Sun L, Chen H, Deng Q, et al. Acquisition of resistance to trastuzumab in gastric cancer cells is associated with activation of IL-6/STAT3/Jagged-1/Notch positive feedback loop. Oncotarget. 2015;6:5072-87.

6. Lewis Phillips GD, Li G, Dugger DL, Crocker LM, Parsons KL, Mai E, et al. Targeting HER2-positive breast cancer with trastuzumab-DM1, an antibody-cytotoxic drug conjugate. Cancer Res. 2008;68:9280-90.

7. Junttila TT, Li G, Parsons K, Phillips GL, Sliwkowski MX. Trastuzumab-DM1 (T-DM1) retains all the mechanisms of action of trastuzumab and efficiently inhibits growth of lapatinib insensitive breast cancer. Breast Cancer Res Treat. 2011;128:347-56.

8. Krop IE, Kim S, González-Martín A, LoRusso PM, Ferrero J, Smitt M, et al. Trastuzumab emtansine versus treatment of physician's choice for pretreated HER2-positive advanced breast cancer (TH3RESA): a randomised, open-label, phase 3 trial. Lancet Oncol. 2014;15:689-99.

9. Wildiers H, Kim S, Gonzalez-Martin A, LoRusso PM, Ferrero $\mathrm{J}$, Yu R, et al. Trastuzumab emtansine improves overall survival versus treatment of physician's choice in patients with previously treated HER2-positive metastatic breast cancer: final overall survival results from the phase 3 TH3RESA study. Cancer Res. 2016;76:Abstract S5-05 (and associated oral presentation).

10. Verma S, Miles D, Gianni L, Krop IE, Welslau M, Baselga J, et al. Trastuzumab emtansine for HER2-positive advanced breast cancer. N Engl J Med. 2012;367:1783-91.

11. Baselga J, Lewis Phillips GD, Verma S, Ro J, Huober J, Guardino AE, et al. Relationship between tumor biomarkers and efficacy in EMILIA, a phase III study of trastuzumab emtansine in HER2-positive metastatic breast cancer. Clin Cancer Res. 2016;22:3755-63.

12. Kim S, Wildiers H, Krop IE, Leung ACF, Trudeau C, Yu R, et al. Relationship between tumor biomarkers (BM) and efficacy in TH3RESA, a phase 3 study of trastuzumab emtansine (T-DM1) versus treatment of physician's choice (TPC) in HER2-positive advanced breast cancer (BC) previously treated with trastuzumab and lapatinib. J Clin Oncol. 2014;32(20 May suppl.):Abstract 605.

13. Thuss-Patience PC, Shah MA, Ohtsu A, Van Cutsem E, Ajani JA, Castro $\mathrm{H}$, et al. Trastuzumab emtansine versus taxane use for previously treated HER2-positive locally advanced or metastatic gastric or gastro-oesophageal junction adenocarcinoma (GATSBY): an international randomised, open-label, adaptive, phase 2/3 study. Lancet Oncol. 2017;18:640-53.

14. Holbro T, Beerli RR, Maurer F, Koziczak M, Barbas CF 3rd, Hynes NE. The ErbB2/ErbB3 heterodimer functions as an 
oncogenic unit: ErbB2 requires ErbB3 to drive breast tumor cell proliferation. Proc Natl Acad Sci USA. 2003;100:8933-8.

15. Baselga J, Swain SM. Novel anticancer targets: revisiting ERBB2 and discovering ERBB3. Nat Rev Cancer. 2009;9:463-75.

16. Cantley LC, Neel BG. New insights into tumor suppression: PTEN suppresses tumor formation by restraining the phosphoinositide 3-kinase/AKT pathway. Proc Natl Acad Sci USA. 1999;96:4240-5.

17. Isakoff SJ, Engelman JA, Irie HY, Luo J, Brachmann SM, Pearline RV, et al. Breast cancer-associated PIK3CA mutations are oncogenic in mammary epithelial cells. Cancer Res. 2005;65:10992-1000.

18. Nagata Y, Lan KH, Zhou X, Tan M, Esteva FJ, Sahin AA, et al. PTEN activation contributes to tumor inhibition by trastuzumab, and loss of PTEN predicts trastuzumab resistance in patients. Cancer Cell. 2004;6:117-27.

19. Eichhorn PJ, Gili M, Scaltriti M, Serra V, Guzman M, Nijkamp W, et al. Phosphatidylinositol 3-kinase hyperactivation results in lapatinib resistance that is reversed by the mTOR/phosphatidylinositol 3-kinase inhibitor NVP-BEZ235. Cancer Res. 2008;68:9221-30.

20. Berns K, Horlings HM, Hennessy BT, Madiredjo M, Hijmans $\mathrm{EM}$, Beelen K, et al. A functional genetic approach identifies the $\mathrm{PI} 3 \mathrm{~K}$ pathway as a major determinant of trastuzumab resistance in breast cancer. Cancer Cell. 2007;12:395-402.

21. Shattuck DL, Miller JK, Carraway KL 3rd, Sweeney C. Met receptor contributes to trastuzumab resistance of Her2-overexpressing breast cancer cells. Cancer Res. 2008;68:1471-7.

22. Chen CT, Kim H, Liska D, Gao S, Christensen JG, Weiser MR. MET activation mediates resistance to lapatinib inhibition of HER2-amplified gastric cancer cells. Mol Cancer Ther. 2012;11:660-9.

23. Baselga J, Cortés J, Im SA, Clark E, Ross G, Kiermaier A, et al. Biomarker analyses in CLEOPATRA: a phase III, placebo-controlled study of pertuzumab in human epidermal growth factor receptor 2-positive, first-line metastatic breast cancer. J Clin Oncol. 2014;32:3753-61.

24. Loibl S, von Minckwitz G, Schneeweiss A, Paepke S, Lehmann A, Rezai M, et al. PIK3CA mutations are associated with lower rates of pathologic complete response to anti-human epidermal growth factor receptor 2 (her2) therapy in primary HER2-overexpressing breast cancer. J Clin Oncol. 2014;32:3212-20.

25. Miller KD, Diéras V, Harbeck N, Andre F, Mahtani RL, Gianni $\mathrm{L}$, et al. Phase IIa trial of trastuzumab emtansine with pertuzumab for patients with human epidermal growth factor receptor 2-positive, locally advanced, or metastatic breast cancer. J Clin Oncol. 2014;32:1437-44.

26. McCarty KS Jr, Szabo E, Flowers JL, Cox EB, Leight GS, Miller $\mathrm{L}$, et al. Use of a monoclonal anti-estrogen receptor antibody in the immunohistochemical evaluation of human tumors. Cancer Res. 1986;46:4244s-8s.
27. Shah MA, Cho JY, Tan IB, Tebbutt NC, Yen CJ, Kang A, et al. A randomized phase II study of FOLFOX with or without the MET inhibitor onartuzumab in advanced adenocarcinoma of the stomach and gastroesophageal junction. Oncologist. 2016;21:1085-90.

28. Bonetti M, Gelber RD. Patterns of treatment effects in subsets of patients in clinical trials. Biostatistics. 2004;5:465-81.

29. Cancer Genome Atlas Research Network. Comprehensive molecular characterization of gastric adenocarcinoma. Nature. 2014;513:202-9.

30. Hofmann M, Stoss O, Shi D, Büttner R, van de Vijver MJ, Kim W, et al. Assessment of a HER2 scoring system for gastric cancer: results from a validation study. Histopathology. 2008;52:797-805.

31. Rüschoff J, Hanna W, Bilous M, Hofmann M, Osamura RY, Penault-Llorca F, et al. HER2 testing in gastric cancer: a practical approach. Mod Pathol. 2012;25:637-50.

32. Van Cutsem E, Bang Y, Feng-yi F, Xu JM, Lee K, Jiao S, et al. HER2 screening data from ToGA: targeting HER2 in gastric and gastroesophageal junction cancer. Gastric Cancer. 2015;18:476-84.

33. Baselga J, Cortés J, Kim SB, Im SA, Hegg R, Im YH, et al. Pertuzumab plus trastuzumab plus docetaxel for metastatic breast cancer. N Engl J Med. 2012;366:109-19.

34. Press MF, Ellis CE, Gagnon RC, Grob TJ, Buyse M, Villalobos I, et al. HER2 status in advanced or metastatic gastric, esophageal, or gastroesophageal adenocarcinoma for entry to the TRIO-013/ LOGiC trial of lapatinib. Mol Cancer Ther. 2017;16:228-38.

35. Satoh T, Xu RH, Chung HC, Sun GP, Doi T, Xu JM, et al. Lapatinib plus paclitaxel versus paclitaxel alone in the second-line treatment of HER2-amplified advanced gastric cancer in Asian populations: TyTAN-a randomized, phase III study. J Clin Oncol. 2014;32:2039-49.

36. Peng Z, Zou J, Zhang X, Yang Y, Gao J, Li Y, et al. HER2 discordance between paired primary gastric cancer and metastasis: a meta-analysis. Chin J Cancer Res. 2015;27:163-71.

37. Seo S, Ryu MH, Ahn JY, Park Y, Park SR, Ryoo BY, et al. Loss of HER2 positivity after anti-HER2 chemotherapy in HER2positive gastric cancer patients: results of GASTric cancer HER2 reassessment study 3 (GASTHER3). J Clin Oncol. 2017;35(4 Suppl):Abstract 27.

38. Esaki T, Makiyama A, Kashiwada T, Hosokawa A, Kawada J, Moriwaki T, et al. T-ACT (WJOG7112G): a randomized phase II study of weekly paclitaxel \pm trastuzumab in patients with HER2positive advanced gastric or gastro-esophageal junction cancer refractory to trastuzumab combined with fluoropyrimidine and platinum. J Clin Oncol. 2017;35(4 Suppl):TPS218.

Publisher's Note Springer Nature remains neutral with regard to jurisdictional claims in published maps and institutional affiliations.

\section{Affiliations}

\section{Manish A. Shah ${ }^{1,10}$ - Yoon-Koo Kang ${ }^{2} \cdot$ Peter C. Thuss-Patience ${ }^{3} \cdot$ Atsushi Ohtsu $^{4} \cdot$ Jaffer A. Ajani ${ }^{5}$. Eric Van Cutsem ${ }^{6} \cdot$ Silke Hoersch $^{7} \cdot$ Marie-Laurence Harle-Yge ${ }^{8} \cdot$ Sanne Lysbet de Haas ${ }^{9}$}

1 Division of Hematology and Medical Oncology, Meyer Cancer Center of Weill Cornell Medical College, New York, NY, USA

2 Department of Oncology, Asan Medical Center, University of Ulsan College of Medicine, Seoul, South Korea
3 Department of Hematology, Oncology and Tumor Immunology, Charité - University Medicine Berlin, Berlin, Germany

4 Department of GI Oncology/Gastroenterology, National Cancer Center Hospital East, Kashiwa, Japan 
5 Department of Gastrointestinal Medical Oncology, University of Texas MD Anderson Cancer Center, Houston, TX, USA

6 Digestive Oncology, University Hospitals Gasthuisberg Leuven and KU Leuven, Leuven, Belgium

7 Statistics Department, KOEHLER eClinical GmbH, Freiburg, Germany
8 Pharmaceutical Development Clinical Oncology, F. Hoffmann-La Roche Ltd, Basel, Switzerland

9 Department of Oncology Biomarker Development, F. Hoffmann-La Roche Ltd, Basel, Switzerland

10 Solid Tumor Service, Gastrointestinal Oncology, Weill Cornell Medicine/New York-Presbyterian Hospital, 1305 York Avenue, New York, NY 10021, USA 